OPEN ACCESS

Edited by:

Tak Fai Joseph Lau, The Chinese University of Hong

Kong, Hong Kong

Reviewed by:

Gerjo Kok,

Maastricht University, Netherlands

Zixin Wang,

The Chinese University of Hong

Kong, Hong Kong

*Correspondence:

Nga Thi Thu Vu

nga.vu@student.unsw.edu.au;

John de Wit

j.dewit@unsw.edu.au

Specialty section:

This article was submitted to

HIV and AIDS,

a section of the journal

Frontiers in Public Health

Received: 02 August 2016 Accepted: 02 December 2016 Published: 19 December 2016

Citation:

Vu NTT, Holt M, Phan HTT, La LT,

Tran GM, Doan TT and de Wit J

(2016) The Prevalence and Correlates of HIV and Undiagnosed Infection among Men Who Have Sex with Men

in Hanoi, Vietnam: Findings from a Cross-sectional, Biobehavioral Study.

Front. Public Health 4:275.

doi: 10.3389/fpubh.2016.00275

\section{The Prevalence and Correlates of HIV and Undiagnosed Infection among Men Who Have Sex with Men in Hanoi, Vietnam: Findings from a Cross-sectional, Biobehavioral Study}

\author{
Nga Thi Thu Vu',2*, Martin Holt', Huong Thi Thu Phan ${ }^{3}$, Lan Thi La4, Gioi Minh Tran ${ }^{5}$, \\ Tung Thanh Doan ${ }^{5}$ and John de Wit ${ }^{1,6 *}$
}

${ }^{1}$ Centre for Social Research in Health, UNSW Australia, Sydney, NSW, Australia, ${ }^{2}$ Institute of Preventive Medicine and Public Health, Hanoi Medical University, Hanoi, Vietnam, ${ }^{3}$ Vietnam Administration for HIVIAIDS Prevention and Control, Ministry of Health, Hanoi, Vietnam, ${ }^{4}$ Hanoi Centre of HIVIAIDS Prevention and Control, Hanoi, Vietnam, ${ }^{5}$ Center for Community Health Promotion, Hanoi, Vietnam, ${ }^{6}$ Utrecht University, Utrecht, Netherlands

Introduction: Men who have sex with men (MSM) are a key population for HIV infection in Vietnam, and the use of amphetamine type substances (ATS) is prevalent and possibly increasing in this population. The reported analysis examines the association between ATS use before or during sex and HIV infection among MSM in Hanoi, Vietnam.

Methods: This cross-sectional study of 210 MSM was conducted in Hanoi, Vietnam, in late 2014. Men tested for HIV and answered questions about demographic characteristics, sexual sensation seeking, depression, belief in HIV prevention strategies, homosexuality-related stigma and discrimination, recent accessing of HIV prevention services, sexual behaviors and ATS, and other drug use behaviors. We performed logistic regression to assess correlates of HIV infection.

Results: HIV prevalence was 6.7\% (14/210), and 85.7\% (12/14) of HIV-positive men were not aware of their HIV status. Of the 210 participants, 10.5, 2.9, and 3.8\% of men had used methamphetamine, amphetamine, and ecstasy before or during sex in the last 3 months. In multivariable analysis, HIV infection was associated with recent sex-related methamphetamine use [adjusted odds ratio (AOR): 5.03, 95\% confidence interval (Cl): 1.35-18.68], engaging in recent sex work (AOR: 3.55, 95\% Cl: 1.07-11.75), and homosexuality-related perceived stigma (AOR: 2.32, 95\% Cl: 0.98-5.47).

Conclusion: Findings underscore the importance of integrating methamphetamine use interventions into HIV prevention services and scaling-up of gay-friendly, non-stigmatizing HIV testing services for MSM in Hanoi. We recommend the routine assessment of ATS use and undiagnosed infection in this population.

Keywords: HIV prevalence, amphetamine type stimulants, men who have sex with men, undiagnosed HIV, Hanoi-Vietnam 


\section{INTRODUCTION}

The HIV epidemic was first described in Vietnam in the early 1990 s (1), and in the last 30 years, the epidemic has spread to different parts of the country. Since 1999, HIV has been reported in all provinces and cities of Vietnam (2). Although men who have sex with men (MSM) have been recognized as a key population affected by HIV in different parts of the world (3), in Vietnam, they were only identified as a priority population for HIV prevention in the last decade (4), despite warning signs in the early 2000s that HIV was spreading in this population $(5,6)$.

A study conducted only in Ho Chi Minh City in 2004 reported an HIV prevalence of $8 \%$ in a sample of MSM recruited in MSM-specific venues (7). The first, national integrated biological behavioral surveillance (IBBS) conducted in 2006 reported that the HIV prevalence among MSM in Ho Chi Minh City and Hanoi was 5 and $9 \%$, respectively (8). In the second IBBS conducted in 2009, HIV prevalence exceeded $10 \%$ in Hanoi $(19.8 \%)$ and Ho Chi Minh City (14.3\%), as well as in Haiphong (16.6\%), the country's third largest city (9), suggesting HIV prevalence had increased substantially among MSM in major cities across Vietnam. A more recent study found an HIV prevalence of $14.8 \%$ among MSM in Ho Chi Minh City (10). A better understanding of the individual, social, and structural factors that affect HIV risks is, therefore, needed to prevent further transmission of HIV in the MSM population in Vietnam.

Previous studies have found that HIV infection among MSM in Vietnam is associated with younger age, lower education and injecting drug use $(7,11)$, a higher number of recent sexual partners $(5,7)$, and sex work and inconsistent condom use (5). MSM in Vietnam have consistently reported low levels of condom use with casual and regular male partners $(8,9)$.

Amphetamine type stimulants (ATS) are commonly used drugs by MSM, and their use has been found to be associated with condomless anal intercourse (CAI) and HIV infection (12). A recent meta-analysis found a significant association between methamphetamine and amphetamine use and HIV infection across 35 studies (13). However, most previous studies have been conducted in high-income countries, and evidence regarding the association between ATS use and HIV infection among MSM is lacking in low- and middle-income countries (13), particularly Vietnam. It was reported that ATS use, particularly methamphetamine use is prevalent among MSM in Vietnam (14). The relationship between ATS use and HIV infection among MSM in Vietnam, however, remains to be assessed.

In Vietnam, homosexuality remains socially stigmatized (15), despite recent activism to protect the rights of lesbian, gay, bisexual, and transgender people. Previous international research underscores that homosexuality-related stigma and discrimination are associated with a higher risk of depression $(16,17)$. Depression has been found to be highly prevalent among MSM, particularly HIV-positive men $(18,19)$, and is considered a major health issue among MSM (20). Furthermore, homosexualityrelated stigma, discrimination, and depression have been found to be associated with engaging in HIV-related risky behaviors such as CAI (21-23), drug use $(24,25)$, having sex while on drugs (26), less awareness and underutilization of HIV prevention services
(27-29), and experiencing less benefit from participating in HIV prevention interventions (27).

Previous studies of HIV infection among MSM in Vietnam have mainly focused on individual-level covariates rather than examining social and structural barriers to HIV prevention, such as homosexuality-related stigma and discrimination. Evidence from previous studies indicates that sexual sensation seeking is associated with an increased likelihood of CAI among MSM (30-33) and that sexual sensation seeking can modify or strengthen the association between alcohol or drug use and HIVrelated sexual behaviors $(34,35)$. However, the effect of sexual sensation seeking on HIV risk among MSM in Vietnam has not been previously investigated.

Previous research in Vietnam has found relatively high rates of CAI between MSM $(8,9)$, and international research has found that MSM may use various strategies to reduce HIV transmission risk during CAI, such as serosorting (having sex with partners of the same perceived HIV status), strategic positioning (HIV-negative men being insertive during CAI), withdrawal before ejaculation, or limiting CAI to HIV-positive partners who are virally suppressed $(36,37)$. However, belief in the efficacy of these strategies has not been previously assessed among Vietnamese MSM.

To improve HIV prevention among MSM in Vietnam, we sought to assess the prevalence of HIV and undiagnosed infection among MSM in Hanoi and examined factors associated with HIV infection, including homosexuality-related stigma and discrimination, depression, sexual sensation seeking, belief in the efficacy of risk reduction strategies, and drug use, particularly ATS use.

\section{MATERIALS AND METHODS}

Participants included in this analysis were part of a larger convenience sample of 303 MSM recruited into a cross-sectional, community-based study in Hanoi, Vietnam, from September to October 2014. The study received approval from the Human Research Ethics Committee of the University of New South Wales, Australia, and from the Institutional Review Board of the Hanoi School of Public Health. A description of the study has been previously published (14). In short, the study was conducted in collaboration with the Hanoi Center for HIV/ AIDS Prevention and Control (Hanoi PAC), the Center for Community Health Promotion (CHP), and MSM communitybased organizations (CBOs). MSM are a relatively hidden population in Vietnam, and developing a randomized sampling frame is impossible. Convenience sampling was hence used to recruit participants. First, staff and outreach workers of collaborating organizations referred potential participants to the study. Additionally, peers of collaborating $\mathrm{CBO}$ referred men from their social networks. Finally, participated men were asked to invite their peers who might be interested in the study.

Men were eligible to participate if they were 18 years or older at the time of the study, reported having anal sex with at least one man in the previous 3 months, had a good command of the Vietnamese language, and provided consent. Men were screened 
for eligibility and were interviewed after provided written consent. Upon interview completion, men were informed about optional HIV testing.

Men who chose to be tested were referred to an on-site testing team provided by Hanoi PAC. Separate written consent was obtained for HIV testing. Venous blood samples were collected by technicians from Hanoi PAC. Blood samples were assigned a unique, anonymous ID code enabling linkage to participants' questionnaires (and for participants to get results). Due to resource constraints, we could only offer 210 free HIV tests to participants.

\section{Confidentiality and Privacy}

Being identified as a MSM or drug user in Vietnam may result in social stigma and discrimination (38). As such, MSM participants, particularly those who use drugs, may underreport sexual and drug use behaviors. We took several steps to mitigate this potential bias. First, all interviewers were trained to be supportive and non-judgmental of MSM. Second, MSM participants were not asked to provide personal details that could reveal their identity. Third, all interviews were conducted in private rooms. Finally, all field staff signed a confidentiality agreement.

\section{HIV Testing and Status}

Blood samples were tested for HIV at the Hanoi PAC laboratory, following Vietnamese Ministry of Health guidelines. All blood samples were screened for HIV by the Murex HIV Ag/ Ab combination assay (DiaSorin S.p.A., Italy). Samples that were reactive during screening were tested again using confirmatory tests (Serodia ${ }^{\circledR}$ HIV, Fujirebio, Japan; Determine ${ }^{\mathrm{TM}}$ HIV1/2, Alere Medical, USA). The main outcome variable was HIV status as confirmed by testing (HIV negative or HIV positive). We also referred to participants' self-reported HIV status (HIV negative, untested/unknown, or HIV positive) to identify participants who tested HIV positive but were unaware of their infection. The self-report information collected through the questionnaire has previously been described (14) and contained the questions described below.

\section{Demographic Characteristics}

We asked participants about their self-reported HIV status, sexual orientation, age, place of birth, education, occupation, and monthly income.

\section{Sexual Sensation Seeking}

We adapted a measure of sexual sensation seeking for a Vietnamese sample (39). Ten items assessed the propensity to seek out exciting and novel sexual experiences, for instance "I like wild, uninhibited sexual encounters." Participants provided their answers on 5-point scales, ranging from 1 (not at all like me) to 4 (very much like me). Internal consistency of the items was sufficient (Cronbach's $\alpha=0.72$ ). Item scores were averaged, with higher scores indicating more sexual sensation seeking.

\section{Depression}

This was assessed with the Patient Health Questionnaire 9 scale, which has been used with MSM in other studies $(40,41)$. The scale consists of nine items, such as "in the last two weeks, how often have you had trouble falling or staying asleep, or sleeping too much?" Participants provided their answers on a scale ranging from 0 (not having the problem at all) to 3 (having the problem nearly every day). The items had good internal consistency (Cronbach's $\alpha=0.80$ ). Participants were categorized as having a depressive disorder if they had a score equal or larger than 10 , as previously described (42).

\section{Belief in HIV Prevention Strategies}

Participants were asked how effective they thought different strategies were in preventing HIV transmission: antiretroviral treatment of HIV, HIV-negative men taking the insertive (top) position during anal sex, and withdrawal before ejaculation. Answer options ranged from 1 (totally disagree) to 4 (totally agree). Belief in the efficacy of each HIV prevention method was dichotomized into disagreement (scores 1 and 2) versus agreement (scores 3 and 4 ).

\section{Perceived Stigma and Discrimination}

We adapted a scale to measure enacted (experienced) homosexuality-related stigma, perceived (anticipated) homosexualityrelated stigma, and self-stigma (internalized homophobia) (22). Responses were given on 4-point scales, with anchors depending on the questions. The adapted scale encompassed eight items pertaining to enacted homosexuality-related stigma, for example "how often have you lost a job or career opportunity due to your engaging in homosexual activities" ( $1=$ never, $4=$ often); 10 items measuring perceived homosexuality-related stigma, for instance "many people are unwilling to accept homosexual individuals" ( $1=$ completely disagree, $4=$ completely agree); and eight items measuring internalized homophobia, for example "sometimes you wish you were not gay/bisexual/transgender" ( 1 = totally disagree, $4=$ totally agree). In this study, the scale had good internal consistency (Cronbach's $\alpha=0.74$ ). Mean scores were calculated for each sub-scale; higher scores indicated higher levels of stigma.

\section{Recent Accessing of HIV Prevention Services}

We asked participants if they had recently tested for HIV (i.e., at least once in the last 12 months) and if they had recently received safe sex counseling (i.e., at least once in the last 12 months).

\section{Sexual Behaviors}

We asked about the gender of sexual partners and age at first sex with men and women. Participants were asked about ever engaging in sex work (selling sex), recent sex work (in the last 3 months), their number of regular and casual male partners in the last 3 and 12 months, and the use of condoms during anal sexual intercourse with regular and casual male partners. Because numbers of different types of sexual partners were skewed, we undertook logarithmic or square root transformations of these variables, as appropriate. Any CAI was defined as not or inconsistently using a condom during anal sex (assessed for regular and casual male partners in the previous 3 months). 


\section{ATS and Other Drug Use}

Participants were asked questions about ever having used ATS, alcohol, and other substances (i.e., ketamine, erectile dysfunction medications, and amyl nitrite "poppers"), any recent use of these substances (in the last 3 months) and any use of these substances before or during sex in the last 3 months. Answers to these questions were dichotomized (any use versus none).

\section{Data Analysis}

Descriptive and correlational statistical analyses were performed using STATA version 13.1 (StataCorp, College Station, TX, USA). We report frequencies and percentages for categorical variables and medians with interquartile ranges (IQR) for continuous variables. We tested bivariate associations between laboratoryconfirmed HIV status and potential covariates using logistic regression. Factors associated with the outcome variable at $p<0.25$ were subsequently entered into a multivariate regression model to identify independent covariates. We used a stepwise procedure as previously suggested (43) to develop the final multivariate model with independent covariates, which retained statistical significance $(p<0.05)$.

\section{RESULTS}

A total of 222 men were referred to the study as potential participants, among whom nine men did not meet selection criteria, two men chose not to complete an interview, and one man refused to be tested. As such, the following analysis includes 210 men who completed an interviewer-administered questionnaire and agreed to be tested for HIV and release their HIV test result. We compared the sociodemographic characteristics of men who did and did not undertake HIV testing (analysis not shown). Men who tested for HIV and consented to release their HIV test results were significantly younger, had lower incomes, and were more likely to be students or self-employed. Men who did and didn't undertake HIV testing were similar in terms of place of birth, education level, sexual orientation, and perceived HIV status.

\section{Sample Characteristics}

Table 1 presents the demographic characteristics of the 210 MSM who participated. The majority of the sample reported being homosexual (73.3\%), 22.4\% bisexual, and the remainder $(3.8 \%)$ heterosexual or other sexual orientation. The median age of the sample was 22.7 years (IQR: 20.6-25.5); 90.0\% were younger than 30 years. The majority of men had a college or university education (57.9\%), 30.1\% had high school or vocational training, and only $12.0 \%$ had secondary or lower education. Approximately $30.0 \%$ of the sample was students, $9.0 \%$ were unemployed, and the remainder had office-based jobs, service jobs, or were in selfemployed, casual, or freelance jobs. Participants had a median monthly income of 5 million Vietnamese Dong (approximately US\$228) (IQR: 3.0-8.0 million). The median sexual sensationseeking score was 2.5 (IQR: 1.4-3.5). A minority of men (14.3\%) were categorized as having depression. In relation to belief in different HIV prevention strategies, 9.5\% men believed in the effectiveness of HIV treatment as prevention, $28.6 \%$ believed in the safety of being insertive during sex, and $38.6 \%$ men believed that withdrawal was effective. The median score regarding homosexuality-related enacted stigma was 1.1 (IQR: 1.0-2.4); scores for perceived stigma and internalized homophobia were 4.0 (IQR: 1.20-5.0) and 3.4 (IQR: 1.0-4.6), respectively.

\section{Sex and Drug Use Behaviors}

Table 2 presents HIV-related sex and drug use behaviors. Approximately $63 \%$ men self-reported having sex with men only; the remainder reported having sex with both men and women. The median age of first homosexual sex was 19.0 (IQR: 18.0-21.0), and the median age of first heterosexual sex was 18.0 (12.0-30.0). Of the 210 participants, $73.8 \%$ reported any recent CAI with male partners $(66.2 \%$ with regular partners and $32.4 \%$ with casual partners). The median number of regular male sexual partners in the last 3 months was 1 (IQR: 1.0-80.0), and the median number of casual male sexual partners in the last 3 months was 3.0 (IQR: $1.0-100.0$ ). Of the 210 participants, $26.2 \%$ reported having ever engaged in sex work and $21.4 \%$ reported recent sex work. One in five participants (22.9\%) reported ever having used methamphetamine, $14.8 \%$ reported recent use, and $10.5 \%$ reported recent sex-related use. The corresponding rates for amphetamine use were 7.6, 2.9 , and $2.9 \%$, respectively. Rates for ecstasy they were 20.0, 8.6 , and $3.8 \%$. Men reported higher levels of alcohol use: $90.0 \%$ had ever drunk alcohol, $76.2 \%$ had recently consumed alcohol, and $39.5 \%$ reported sex-related alcohol use in the last 3 months.

\section{HIV Testing Results}

Fourteen men out of 210 [6.7\%, 95\% confidence interval (CI): 3.7-10.9] tested HIV-antibody positive (see Table 1) and 12 of these 14 HIV-positive men (85.7\%) were not aware of their HIV status. Just over half (52.4\%) of all participating men had ever tested for HIV, and just under half (46.6\%) did not know their HIV status, including 29.5\% who had ever tested for HIV but did not know their test result and $17.1 \%$ who had never tested for HIV. Out of the 210 men who were tested, approximately 10 men returned for their results, including one man who was previously undiagnosed with HIV.

\section{Correlates of HIV Infection}

The results of bivariate and multivariate analyses of associations between HIV infection and other covariates are presented in the Table 3. Potential independent covariates identified in bivariate analysis included occupation, sexual sensation seeking, belief in the safety of being insertive during sex as a HIV prevention strategy, homosexuality-related perceived stigma, engaging in recent sex work, number of regular male sexual partners, any recent CAI with casual male sexual partners, recent methamphetamine or amphetamine use before or during sex, and having a HIV test in the last 12 months. In the final multivariate logistic regression model, HIV infection was more likely among MSM who reported recent sex work or the use of methamphetamine before or during sex. Additionally, HIV infection was marginally associated with homosexualityrelated perceived stigma. 
TABLE 1 | Participant characteristics.

\begin{tabular}{|c|c|c|}
\hline & Frequency & Percent $(95 \% \mathrm{Cl})$ \\
\hline \multicolumn{3}{|l|}{ HIV status confirmed by lab tests $(N=210)$} \\
\hline Negative & 196 & $93.3(89.1-96.3)$ \\
\hline Positive & 14 & $6.7(3.7-10.9)$ \\
\hline \multicolumn{3}{|l|}{ Perceived HIV status (on enrollment) $(N=210)$} \\
\hline HIV positive & 2 & $0.9(0.1-3.4)$ \\
\hline HIV negative & 110 & $52.4(45.4-59.3)$ \\
\hline Tested but didn't know the result & 62 & $29.5(23.4-36.2)$ \\
\hline Never previously tested & 36 & $17.1(12.3-22.9)$ \\
\hline \multicolumn{3}{|l|}{ Sexual identity $(N=210)$} \\
\hline Homosexual & 154 & $73.3(66.8-79.2)$ \\
\hline Bisexual & 47 & $22.4(16.9-28.6)$ \\
\hline Heterosexual and other & 9 & $4.3(2.0-8.0)$ \\
\hline Age [median and interquartile ranges (IQR)] $(N=210)$ & $22.7(20.6-25.5)$ & \\
\hline$<20$ & 38 & $18.1(13.1-24.0)$ \\
\hline 20-29 & 151 & $71.9(65.3-77.9)$ \\
\hline 30-39 & 14 & $6.7(3.7-10.9)$ \\
\hline$\geq 40$ & 7 & $3.3(1.4-6.7)$ \\
\hline \multicolumn{3}{|l|}{ Place of birth $(N=210)$} \\
\hline Hanoi & 86 & $41.0(34.2-47.9)$ \\
\hline Other provinces & 124 & $59.0(52.1-65.8)$ \\
\hline \multicolumn{3}{|l|}{ Education $(N=209)$} \\
\hline Primary and lower school & 25 & $12.0(7.9-17.1)$ \\
\hline High school and vocational training & 63 & $30.1(24.0-36.9)$ \\
\hline College and university & 121 & $57.9(50.9-64.7)$ \\
\hline \multicolumn{3}{|l|}{ Occupation $(N=210)$} \\
\hline Student & 66 & $31.4(25.2-38.2)$ \\
\hline Office-based job & 35 & 16.7 (11.9-22.4) \\
\hline Service job & 38 & $18.1(13.1-24.0)$ \\
\hline Self-employed/freelance & 52 & $24.8(19.1-31.2)$ \\
\hline Unemployed & 19 & $9.0(5.5-13.8)$ \\
\hline Median monthly income (IQR) $(N=210)$ & $5.0(3.0-8.0)$ & \\
\hline Income $<3$ million VND & 37 & $17.6(12.7-23.5)$ \\
\hline 3 million VND $\leq$ income $<5$ million VND & 62 & $29.5(23.4-36.2)$ \\
\hline Income $\geq 5$ million VND & 111 & $52.9(45.9-59.8)$ \\
\hline Median sexual sensation-seeking score $(I Q R)(N=210)$ & $2.5(1.4-3.5)$ & \\
\hline Depression $(N=210)$ & 30 & $14.3(9.9-17.8)$ \\
\hline \multicolumn{3}{|l|}{ Belief in HIV prevention strategies $(N=210)$} \\
\hline Treatment as prevention & 20 & $9.5(5.9-14.3)$ \\
\hline Being insertive during anal sex & 60 & $28.6(22.6-35.2)$ \\
\hline Withdrawal & 81 & $38.6(32.0-45.5)$ \\
\hline \multicolumn{3}{|c|}{ Median score for homosexuality-related stigma and discrimination (IQR) $(N=210)$} \\
\hline Enacted stigma & $1.1(1.0-2.4)$ & \\
\hline Perceived stigma & $4.0(1.2-5.0)$ & \\
\hline Internalized homophobia & $3.4(1.0-4.6)$ & \\
\hline Any HIV test in the last 12 months $(N=210)$ & 93 & $44.3(37.5-51.3)$ \\
\hline Received any safe sex counseling in the last 12 months $(N=210)$ & 110 & $52.4(45.4-59.3)$ \\
\hline
\end{tabular}

\section{DISCUSSION}

To the best of our knowledge, this is the first reported analysis of an association between a broad range of important structural, personal dispositions, and behavioral factors and HIV infection among MSM in Vietnam. While we found that MSM who reported recently using amphetamine or ecstasy before or during sex were not more likely to have an HIV positive test result, MSM who self-reported the recent use of methamphetamine before or during sex had a fivefold increased likelihood of HIV infection, compared with MSM who did not use methamphetamine for sex. These findings are similar to those of a recent meta-analysis (13), as well as recent empirical research, finding higher rates of methamphetamine and/or amphetamine use among diagnosed HIV-positive MSM compared to HIV-negative MSM (44-47). In our study, we also found that men who engaged in recent sex work were more likely to test positive for HIV. Together, our findings suggest that men who use ATS for sex and men who engage in sex work should be targeted as priority groups for HIV prevention in Hanoi, Vietnam. We also suggest that interventions for methamphetamine use are made available for MSM in Vietnam and be incorporated into current HIV prevention activities. 
TABLE 2 | Sexual and drug use behaviors.

\begin{tabular}{|c|c|c|}
\hline & Frequency & Percent $(95 \% \mathrm{Cl})$ \\
\hline \multicolumn{3}{|l|}{ Type of sexual partners $(N=210)$} \\
\hline Male sexual partners only & 132 & $62.9(55.9-69.4)$ \\
\hline Both male and female sexual partners & 78 & $37.1(30.6-44.1)$ \\
\hline Median age at first homosexual sex $(N=210)(I Q R)$ & $19.0(18.0-21.0)$ & \\
\hline Median age at first heterosexual sex $(N=78)(I Q R)$ & $18.0(12.0-30.0)$ & \\
\hline Any condomless anal intercourse (CAI) with male partners in the last 3 months $(N=210)$ & 155 & $73.8(67.3-79.6)$ \\
\hline \multicolumn{3}{|l|}{ Regular male sexual partners } \\
\hline Median number of regular male sexual partners in the last 3 months ( $N=196)(I Q R)$ & $1(1-80)$ & \\
\hline Any CAI with regular male sexual partners in the last 3 months $(N=210)$ & 139 & $66.2(59.4-72.6)$ \\
\hline \multicolumn{3}{|l|}{ Casual male sexual partners } \\
\hline Median number of casual male sexual partners in the last 3 months $(N=128)(I Q R)$ & $3(1-100)$ & \\
\hline Any CAI with casual male sexual partners in the last 3 months $(N=210)$ & 68 & $32.4(26.1-39.1)$ \\
\hline Ever engaged in sex work $(\mathrm{N}=210)$ & 55 & $26.2(20.4-32.7)$ \\
\hline Engaged in sex work in the last 3 months $(N=210)$ & 45 & $21.4(16.1-27.6)$ \\
\hline \multicolumn{3}{|l|}{ Lifetime use of alcohol and other drugs $(N=210)$} \\
\hline Alcohol & 189 & $90.0(85.1-93.7)$ \\
\hline Amphetamine (speed) & 16 & $7.6(4.4-12.1)$ \\
\hline Amyl nitrite (poppers) & 11 & $5.2(2.6-9.2)$ \\
\hline Cannabis & 35 & $16.7(11.9-22.4)$ \\
\hline Ecstasy & 42 & $20.0(14.8-26.1)$ \\
\hline Erectile dysfunction medication & 15 & $7.1(4.1-11.5)$ \\
\hline Heroin & 12 & $5.7(3.0-9.8)$ \\
\hline Ketamine & 16 & $7.6(4.4-12.1)$ \\
\hline Methamphetamine & 48 & $22.9(17.4-29.1)$ \\
\hline Sleeping pills & 8 & $3.8(1.7-7.4)$ \\
\hline \multicolumn{3}{|l|}{ Any alcohol and ATS use in the last 3 months $(N=210)$} \\
\hline Alcohol & 160 & $76.2(69.8-81.8)$ \\
\hline Amphetamine (speed) & 6 & $2.9(1.1-6.1)$ \\
\hline Ecstasy & 18 & $8.6(5.2-13.2)$ \\
\hline Methamphetamine & 31 & $14.8(10.3-20.3)$ \\
\hline \multicolumn{3}{|l|}{ Any alcohol and ATS use before or during sex in the last 3 months $(N=210)$} \\
\hline Alcohol & 83 & $39.5(32.9-46.4)$ \\
\hline Amphetamine (speed) & 6 & $2.9(1.1-6.1)$ \\
\hline Ecstasy & 8 & $3.8(1.7-7.4)$ \\
\hline Methamphetamine & 22 & $10.5(6.7-15.4)$ \\
\hline
\end{tabular}

$C l$, confidence interval; IQR, interquartile range.

Furthermore, future periodic surveillance of HIV among MSM in Vietnam would benefit from the inclusion of measures of ATS use and its association with sexual risk behaviors. Such research would contribute to an increasing understanding of the complex relationships between drug use, sexual behaviors, and HIV infection among Vietnamese MSM that can guide HIV prevention.

We found a relatively moderate prevalence of HIV (6.7\%) among MSM recruited in Hanoi, lower than that found in previous government-run surveillance studies $(6,8)$. Our convenience sample did not include any MSM who reported injecting drug use, so we may have under-recruited men who are at higher risk of HIV. As such, generalizing our findings to the broader community of MSM in Hanoi should therefore be undertaken with caution. Strikingly, we found the majority of HIV-positive men (12 out of 14) indicated that they were HIV-negative or did not know their HIV status. To the best of our knowledge, no previous studies have reported the level of undiagnosed HIV among MSM in Vietnam $(7,9,11,48)$, and our results suggest that many HIV-positive MSM in Hanoi may be unaware of their infection. Some of the HIV-positive men in our study may have chosen not to reveal that they had already been diagnosed when interviewed, because of reticence or fear about disclosing their status. Previous studies in high-income countries, while reporting lower levels of undiagnosed HIV $(49,50)$, have found that men with undiagnosed HIV report more risky sexual and drug use behaviors than HIV-negative men (49). We also found a borderline association between homosexuality-related perceived stigma and HIV infection in our analysis. Therefore, promoting accessible HIV testing services, for example, MSM-run, community-based, HIV testing services, is recommended, because they could encourage HIV testing as well as returning for HIV test results (51). Additionally, as little is known about undiagnosed HIV among MSM in Vietnam, future research could assess the extent of undiagnosed HIV and its correlates in different parts of the country.

We did not find a significant association between HIV infection and any form of CAI, despite previous research establishing CAI as a key risk factor for HIV infection in MSM (52). Since the sample size for this analysis was relatively small, power may have been insufficient to detect an association between CAI and HIV infection. Alternatively, the lack of variance between HIV status 
TABLE 3 | Bivariate and multivariate analysis of associations with HIV infection $(N=210)$.

\begin{tabular}{|c|c|c|c|c|}
\hline & COR $(95 \% \mathrm{Cl})$ & $p$ & AOR $(95 \% \mathrm{Cl})$ & $p$ \\
\hline Age group (in years) & & 0.415 & & \\
\hline$<20$ & 1 & & & \\
\hline $20-29$ & $1.14(0.24-5.51)$ & 0.87 & & \\
\hline$\geq 30$ & $3.0(0.46-19.59)$ & 0.251 & & \\
\hline Education & & 0.01 & & 0.843 \\
\hline Primary and lower school only & 1 & & 1 & \\
\hline High school and above & $5.61(1.52-20.79)$ & & $1.20(0.20-7.34)$ & \\
\hline Occupation & & 0.04 & & \\
\hline Student or office-based job & 1 & & 1 & \\
\hline Service job & $4.95(1.12-21.85)$ & 0.035 & $0.30(0.01-8.65)$ & 0.485 \\
\hline Self-employed/freelance & $4.26(1.02-17.80)$ & 0.047 & $0.28(0.01-6.82)$ & 0.435 \\
\hline Unemployed & 1 (omitted) $)^{\mathrm{a}}$ & & & \\
\hline Monthly income & & 0.307 & & \\
\hline <3 million VND (US\$163.4) & 1 & & & \\
\hline 3 million-4.9 million VND & $0.28(0.05-1.58)$ & 0.148 & & \\
\hline$\geq 5$ million VND & $0.64(0.18-2.26)$ & 0.49 & & \\
\hline Sexual orientation & & 0.857 & & \\
\hline Homosexual & 1 & & & \\
\hline Bisexual & $0.89(0.24-3.32)$ & 0.858 & & \\
\hline Heterosexual and other & 1 (omitted) $^{\mathrm{a}}$ & & & \\
\hline Had major depression in the last 2 weeks & $2.56(0.51-12.74)$ & 0.252 & & \\
\hline Sexual sensation seeking & $4.59(1.17-17.96)$ & 0.023 & $2.27(0.47-11.08)$ & 0.31 \\
\hline Belief in the safety of withdrawal as a prevention strategy & $0.88(0.28-2.72)$ & 0.82 & & \\
\hline Belief in the effectiveness of HIV treatment as a prevention strategy & 1 (omitted) $^{\mathrm{a}}$ & & & \\
\hline Belief in the safety of being insertive during sex as a prevention strategy & $0.40(0.09-1.83)$ & 0.235 & $0.25(0.05-1.32)$ & 0.102 \\
\hline Homosexuality-related enacted stigma & $1.48(0.24-9.20)$ & 0.672 & & \\
\hline Homosexuality-related perceived stigma & $1.99(0.92-4.31)$ & 0.081 & $2.32(0.98-5.47)$ & 0.054 \\
\hline Homosexuality-related homophobia & $0.78(0.43-1.42)$ & 0.425 & & \\
\hline Had male partners only versus both male and female partners & $0.94(0.30-2.90)$ & 0.909 & & \\
\hline Engaged in sex work (selling sex) in the last 3 months & $4.16(1.38-12.56)$ & 0.012 & $3.55(1.07-11.75)$ & 0.038 \\
\hline Number of regular male sexual partners & $0.24(0.04-1.63)$ & 0.145 & $1.38(0.08-23.31)$ & 0.822 \\
\hline Number of casual male sexual partners & $1.34(0.81-2.20)$ & 0.255 & & \\
\hline Any condomless anal intercourse (CAI) with regular sexual partners in the last 3 months & $1.95(0.53-7.22)$ & 0.319 & & \\
\hline Any CAI with casual sexual partners in the last 3 months & $3.02(1.00-9.09)$ & 0.049 & $1.76(0.29-10.58)$ & 0.537 \\
\hline Any $\mathrm{CAl}$ in the last 3 months & $1.32(0.36-4.93)$ & 0.676 & & \\
\hline Methamphetamine use before or during sex in the last 3 months & $5.85(1.76-19.44)$ & 0.004 & $5.03(1.35-18.68)$ & 0.016 \\
\hline Amphetamine use before or during sex in the last 3 months & $8.0(1.33-48.15)$ & 0.023 & $1.73(0.14-21.29)$ & 0.668 \\
\hline Ecstasy use before or during sex in the last 3 months & 1 (omitted)a $^{a}$ & & & \\
\hline Drinking alcohol before or during sex in the last 3 months & $1.58(0.53-4.68)$ & 0.41 & & \\
\hline Having at least one HIV test in the last 12 months & $0.48(0.15-1.59)$ & 0.229 & $1.0(0.21-4.68)$ & 0.999 \\
\hline Receiving any safe sex counseling in the last 12 months & $0.66(0.22-1.98)$ & 0.463 & & \\
\hline
\end{tabular}

COR, crude odds ratio; AOR, adjusted odds ratio.

ane of the cells contained a value of $O$ and was excluded from the analysis.

groups may reflect a ceiling effect of high levels of CAI in both HIV-positive and non-HIV-positive MSM. Because CAI with male sexual partners was so common in the sample (73.8\%) and the level of undiagnosed HIV was strikingly high, we recommend an intensified promotion of safe sex among MSM in Hanoi.

Previous studies have found that depression can be highly prevalent among MSM and is more likely higher among diagnosed, HIV-positive MSM $(41,53)$. However, we did not find an association between depression and HIV infection in our sample. This may be because we found a relatively low prevalence of depression in the sample, and the majority of men who had HIV were unaware of their infection. We also did not find an independent association between sexual sensation seeking and HIV infection. However, like other researchers, we have previously found a positive relationship between sexual sensation seeking and CAI (30-33). 
Our study had several limitations. Since MSM are a hidden population in Vietnam, we used a convenience sampling approach, as a result of which our findings may not be representative of the broader MSM population in Hanoi. Our sample is more likely to be representative of young MSM in urban settings in Vietnam who are students or self-employed and have lower incomes. As MSM are a hidden population in Vietnam, previous studies have also used a variety of nonrandom convenience sampling methods and, as in many high income countries, no randomized samples of MSM have been recruited in Vietnam $(8,9,38,54-56)$. Also, although we tried to eliminate reporting bias during the interview process, our findings might reflect underreporting of sexual and drug use behaviors and other socially sensitive issues. Additionally, men who engaged in transactional sex were disproportionately affected by HIV (57). As one fifth of the sample engaged in sex work, assessing sex practices with their sexual partners would have been useful but no such assessment was included in our study.

\section{CONCLUSION}

We found a moderate prevalence of HIV infection among MSM in Hanoi, Vietnam, and most of the men who tested HIV-positive seemed to be unaware of infection. HIV infection was associated with sex-related methamphetamine use and engagement in sex work. Our analytical and descriptive findings point to several recommendations. For HIV prevention, we recommend an integration of interventions for methamphetamine use into HIV prevention, an intensified promotion of safe sex, and implementation of community-based, MSM-run, or MSM-friendly HIV testing services in Hanoi, Vietnam. In research, we recommend the routine assessment of ATS use in national HIV surveillance and research to enable analysis of trends in ATS use and associations with sexual behaviors. We also recommend further study of

\section{REFERENCES}

1. Nguyen TH, WolffersI.HIV infection in Vietnam. Lancet(1994)343(8894):410. doi:10.1016/S0140-6736(94)91239-4

2. Quan VM, Chung A, Long HT, Dondero TJ. HIV in Vietnam: the evolving epidemic and the prevention response, 1996 through 1999. J Acquir Immune Defic Syndr (2000) 25(4):360-9. doi:10.1097/00126334-20001201000011

3. Sullivan PS, Hamouda O, Delpech V, Geduld JE, Prejean J, Semaille C, et al. Reemergence of the HIV epidemic among men who have sex with men in North America, Western Europe, and Australia, 1996-2005. Ann Epidemiol (2009) 19(6):423-31. doi:10.1016/j.annepidem.2009.03.004

4. Socialist Republic of Vietnam. Vietnam AIDS Response Progress Report 2014. Following up the 2011 Political Declaration on HIV/AIDS. Hanoi, Vietnam (2014).

5. Colby D, Cao NH, Doussantousse S. Men who have sex with men and HIV in Vietnam: a review. AIDS Educ Prev (2004) 16(1):45-54. doi:10.1521/ aeap.16.1.45.27722

6. Garcia MC, Meyer SB, Ward P. Elevated HIV prevalence and risk behaviours among men who have sex with men (MSM) in Vietnam: a systematic review. BMJ Open (2012) 2(5). doi:10.1136/bmjopen-2012-001511

7. Nguyen TA, Nguyen HT, Le GT, Detels R. Prevalence and risk factors associated with HIV infection among men having sex with men in Ho Chi Minh City, Vietnam. AIDS Behav (2008) 12(3):476-82. doi:10.1007/s10461-0079267-y men's beliefs and practices with respect to various harm reduction strategies. Finally, we urge examination of the extent of undiagnosed HIV infection in MSM in different parts of Vietnam.

\section{AUTHOR CONTRIBUTIONS}

NV led the development of the research protocol and data collection tools, undertook data collection and data analysis, wrote the initial draft of the manuscript, and prepared the final manuscript. JW and $\mathrm{MH}$ guided and supervised the development of the research protocol, data collection and analysis, and contributed to the writing of the manuscript. HP, LL, GT, and TD provided advice and guidance on the research protocol and data collection and contributed to the manuscript.

\section{ACKNOWLEDGMENTS}

We acknowledge the great support and input from staff of the Institute of Preventive Medicine and Public Health, Hanoi Medical University, Hanoi Centre of HIV/AIDS Prevention and Control, Center for Community Health Promotion, Center for Promotion of Quality of Life and leaders and representatives of community-based organizations of MSM in Hanoi during the data collection process.

\section{FUNDING}

NV was supported by an Australia Awards Scholarship to study at UNSW Australia. The study received financial support from the Faculty of Arts and Social Sciences, UNSW Australia. The Centre for Social Research in Health is supported by the Australian Government Department of Health. Funding agencies were not involved in the development of the research protocol, data collection, data analysis, interpretation, or the dissemination of research findings.

8. Vietnam Ministry of Health. Results from the HIV/STI Intergrated Biological and Behavioural Surveillance (IBBS) in Vietnam, 2005-2006. Hanoi, Vietnam: Ministry of Health (2006).

9. Vietnam Ministry of Health. Results from the HIV/STI Biological and Behavioral Surveillance (Ibbs) in Vietnam - Round II 2009. Hanoi, Vietnam: Ministry of Health (2011).

10. Le TM, Lee PC, Stewart DE, Long TN, Quoc CN. What are the risk factors for HIV in men who have sex with men in Ho Chi Minh City, Vietnam? A cross-sectional study. BMC Public Health (2016) 16(1):406. doi:10.1186/ s12889-016-3088-8

11. Pham QD, Nguyen TV, Hoang CQ, Cao V, Khuu NV, Phan HT, et al. Prevalence of HIV/STIs and associated factors among men who have sex with men in An Giang, Vietnam. Sex Transm Dis (2012) 39(10):799-806. doi:10.1097/ OLQ.0b013e318265b180

12. Colfax G, Santos GM, Chu P, Vittinghoff E, Pluddemann A, Kumar S, et al. Amphetamine-group substances and HIV. Lancet (2010) 376(9739):458-74. doi:10.1016/S0140-6736(10)60753-2

13. Thu Vu NT, Maher L, Zablotska I. Amphetamine-type stimulants and HIV infection among men who have sex with men: implications on HIV research and prevention from a systematic review and meta-analysis. J Int AIDS Soc (2015) 18(1):19273. doi:10.7448/IAS.18.1.19273

14. Vu NT, Holt M, Phan HT, Le HT, La LT, Tran GM, et al. Amphetamine-type stimulant use among men who have sex with men (MSM) in Vietnam: results from a socio-ecological, community-based study. Drug Alcohol Depend (2016) 158:110-7. doi:10.1016/j.drugalcdep.2015.11.016 
15. Horton P. 'I thought I was the only one': the misrecognition of LGBT youth in contemporary Vietnam. Cult Health Sex (2014) 16(8):960-73. doi:10.1080/ 13691058.2014 .924556

16. Secor AM, Wahome E, Micheni M, Rao D, Simoni JM, Sanders EJ, et al. Depression, substance abuse and stigma among men who have sex with men in coastal Kenya. AIDS (2015) 29(Suppl 3):S251-9. doi:10.1097/ QAD.0000000000000846

17. Mills TC, Paul J, Stall R, Pollack L, Canchola J, Chang YJ, et al. Distress and depression in men who have sex with men: the Urban Men's Health Study. Am J Psychiatry (2004) 161(2):278-85. doi:10.1176/appi.ajp.161.2.278

18. Klein H. Depression and HIV risk taking among men who have sex with other men (MSM) and who use the internet to find partners for unprotected sex. J Gay Lesbian Ment Health (2014) 18(2):164-89. doi:10.1080/19359705.2013. 834858

19. Fendrich M, Avci O, Johnson TP, Mackesy-Amiti ME. Depression, substance use and HIV risk in a probability sample of men who have sex with men. Addict Behav (2013) 38(3):1715-8. doi:10.1016/j.addbeh.2012.09.005

20. Gee R. Primary care health issues among men who have sex with men. J Am Acad Nurse Pract (2006) 18(4):144-53. doi:10.1111/j.1745-7599.2006. 00117.x

21. Ross MW, Berg RC, Schmidt AJ, Hospers HJ, Breveglieri M, Furegato M, et al. Internalised homonegativity predicts HIV-associated risk behavior in European men who have sex with men in a 38-country cross-sectional study: some public health implications of homophobia. BMJ Open (2013) 3(2):e001928. doi:10.1136/bmjopen-2012-001928

22. Deuba K, Ekstrom AM, Shrestha R, Ionita G, Bhatta L, Karki DK. Psychosocial health problems associated with increased HIV risk behavior among men who have sex with men in Nepal: a cross-sectional survey. PLoS One (2013) 8(3):e58099. doi:10.1371/journal.pone.0058099

23. De Santis JP, Colin JM, Provencio Vasquez E, McCain GC. The relationship of depressive symptoms, self-esteem, and sexual behaviors in a predominantly Hispanic sample of men who have sex with men. Am J Mens Health (2008) 2(4):314-21. doi:10.1177/1557988307312883

24. Lee JH, Gamarel KE, Bryant KJ, Zaller ND, Operario D. Discrimination, mental health, and substance use disorders among sexual minority populations. LGBT Health (2016) 3(4):258-65. doi:10.1089/lgbt.2015.0135

25. Darke S, Kaye S, McKetin R, Duflou J. Major physical and psychological harms of methamphetamine use. Drug Alcohol Rev (2008) 27(3):253-62. doi:10.1080/09595230801923702

26. Diaz RM, Ayala G, Bein E. Sexual risk as an outcome of social oppression: data from a probability sample of Latino gay men in three U.S. cities. Cultur Divers Ethnic Minor Psychol (2004) 10(3):255-67. doi:10.1037/1099-9809.10. 3.255

27. Huebner DM, Davis MC, Nemeroff CJ, Aiken LS. The impact of internalized homophobia on HIV preventive interventions. Am J Community Psychol (2002) 30(3):327-48. doi:10.1023/A:1015325303002

28. Andrinopoulos K, Hembling J, Guardado ME, de Maria Hernández F, Nieto AI, Melendez G. Evidence of the negative effect of sexual minority stigma on HIV testing among MSM and transgender women in San Salvador, El Salvador. AIDS Behav (2015) 19(1):60-71. doi:10.1007/s10461-014-0813-0

29. O'Cleirigh C, Newcomb ME, Mayer KH, Skeer M, Traeger L, Safren SA. Moderate levels of depression predict sexual transmission risk in HIVinfected MSM: a longitudinal analysis of data from six sites involved in a "prevention for positives" study. AIDS Behav (2013) 17(5):1764-9. doi:10.1007/ s10461-013-0462-8

30. Vu NT, Holt M, Phan HT, La LT, Tran GM, Doan TT, et al. The relationship between methamphetamine use, sexual sensation seeking and condomless anal intercourse among men who have sex with men in Vietnam: results of a community-based, cross-sectional study. AIDS Behav (2016). doi:10.1007/ s10461-016-1467-x

31. Chng CL, Geliga-Vargas J. Ethnic identity, gay identity, sexual sensation seeking and HIV risk taking among multiethnic men who have sex with men. AIDS Educ Prev (2000) 12(4):326-39.

32. Kalichman SC, Heckman T, Kelly JA. Sensation seeking as an explanation for the association between substance use and HIV-related risky sexual behavior. Arch Sex Behav (1996) 25(2):141-54. doi:10.1007/BF0 2437933

33. Kalichman SC, Simbayi L, Jooste S, Vermaak R, Cain D. Sensation seeking and alcohol use predict HIV transmission risks: prospective study of sexually transmitted infection clinic patients, Cape Town, South Africa. Addict Behav (2008) 33(12):1630-3. doi:10.1016/j.addbeh.2008.07.020

34. Heidinger B, Gorgens K, Morgenstern J. The effects of sexual sensation seeking and alcohol use on risky sexual behavior among men who have sex with men. AIDS Behav (2015) 19(3):431-9. doi:10.1007/s10461-014-0871-3

35. Newcomb ME, Clerkin EM, Mustanski B. Sensation seeking moderates the effects of alcohol and drug use prior to sex on sexual risk in young men who have sex with men. AIDS Behav (2011) 15(3):565-75. doi:10.1007/ s10461-010-9832-7

36. Parsons JT, Schrimshaw EW, Wolitski RJ, Halkitis PN, Purcell DW, Hoff CC, et al. Sexual harm reduction practices of HIV-seropositive gay and bisexual men: serosorting, strategic positioning, and withdrawal before ejaculation. AIDS (2005) 19(Suppl 1):S13-25. doi:10.1097/01.aids.0000167348. $15750.9 \mathrm{a}$

37. Holt M, Lea T, Mao L, Zablotska I, Prestage G, de Wit J. Brief report: HIV prevention by Australian gay and bisexual men with casual partners: the emergence of undetectable viral load as one of a range of risk reduction strategies. J Acquir Immune Defic Syndr (2015) 70(5):545-8. doi:10.1097/ QAI.0000000000000787

38. Ha H, Risser JM, Ross MW, Huynh NT, Nguyen HT. Homosexualityrelated stigma and sexual risk behaviors among men who have sex with men in Hanoi, Vietnam. Arch Sex Behav (2015) 44(2):349-56. doi:10.1007/ s10508-014-0450-8

39. Kalichman SC, Rompa D. Sexual sensation seeking and Sexual Compulsivity Scales: reliability, validity, and predicting HIV risk behavior. J Pers Assess (1995) 65(3):586-601. doi:10.1207/s15327752jpa6503_16

40. Mao L, Kidd MR, Rogers G, Andrews G, Newman CE, Booth A, et al. Social factors associated with major depressive disorder in homosexually active, gay men attending general practices in urban Australia. Aust N Z J Public Health (2009) 33(1):83-6. doi:10.1111/j.1753-6405.2009.00344.x

41. Hirshfield S, Wolitski RJ, Chiasson MA, Remien RH, Humberstone M, Wong T. Screening for depressive symptoms in an online sample of men who have sex with men. AIDS Care (2008) 20(8):904-10. doi:10.1080/ 09540120701796892

42. Kroenke K, Spitzer RL, Williams JB. The PHQ-9: validity of a brief depression severity measure. J Gen Intern Med (2001) 16(9):606-13. doi:10.1046/ j.1525-1497.2001.016009606.x

43. Hosmer DW. Chapter 4: Model-building strategies and methods for logistic regression. In: Lemeshow S, Sturdivant RX, editors. Applied Logistic Regression. 3rd ed. Hoboken, NJ: Wiley (2013). p. 89-151.

44. Morineau G, Nugrahini N, Riono P, Nurhayati, Girault P, Mustikawati DE, et al. Sexual risk taking, STI and HIV prevalence among men who have sex with men in six Indonesian cities. AIDS Behav (2011) 15(5):1033-44. doi:10.1007/s10461-009-9590-6

45. Lyons A, Pitts M, Grierson J. Methamphetamine use in a nationwide online sample of older Australian HIV-positive and HIV-negative gay men. Drug Alcohol Rev (2013) 32(6):603-10. doi:10.1111/dar.12072

46. Truong HM, Kellogg TA, McFarland W, Louie B, Klausner JD, Philip SS, et al. Sentinel surveillance of HIV-1 transmitted drug resistance, acute infection and recent infection. PLoS One (2011) 6(10):e25281. doi:10.1371/journal. pone. 0025281

47. Kral AH, Lorvick J, Ciccarone D, Wenger L, Gee L, Martinez A, et al. HIV prevalence and risk behaviors among men who have sex with men and inject drugs in San Francisco. J Urban Health (2005) 82(1 Suppl 1):i43-50. doi:10.1093/jurban/jti023

48. Nguyen TV, Van Khuu N, Nguyen PD, Tran HP, Phan HT, Phan LT, et al. Sociodemographic factors, sexual behaviors, and alcohol and recreational drug use associated with HIV among men who have sex with men in Southern Vietnam. AIDS Behav (2016) 20(10):2357-71. doi:10.1007/s10461-015-1265-x

49. Holt M, Lea T, Asselin J, Hellard M, Prestage G, Wilson D, et al. The prevalence and correlates of undiagnosed HIV among Australian gay and bisexual men: results of a national, community-based, bio-behavioural survey. J Int AIDS Soc (2015) 18:20526. doi:10.7448/IAS.18.1.20526

50. Ferrer L, Furegato M, Foschia JP, Folch C, Gonzalez V, Ramarli D, et al. Undiagnosed HIV infection in a population of MSM from six European cities: results from the Sialon project. Eur J Public Health (2015) 25(3):494-500. doi:10.1093/eurpub/cku139

51. World Health Organization (WHO). Consolidated Guidelines on HIV Testing Services. Geneva, Switzerland: World Health Organization (2015). 
52. Beyrer C. HIV epidemiology update and transmission factors: risks and risk contexts - 16th International AIDS Conference epidemiology plenary. Clin Infect Dis (2007) 44(7):981-7. doi:10.1086/512371

53. Meyer IH. Prejudice, social stress, and mental health in lesbian, gay, and bisexual populations: conceptual issues and research evidence. Psychol Bull (2003) 129(5):674-97. doi:10.1037/0033-2909.129.5.674

54. National Institute of Hygiene and Epidemioloty (Vietnam Ministry of Health). HIV/STI Intergrated Biological and Behavioural Surveillance (IBBS) in Vietnam. Results from Round III and Trends Across Three Round (20052009-2013) of Survey. Hanoi, Vietnam: National Institute of Hygiene and Epidemiology (2014).

55. Bengtsson L, Lu X, Liljeros F, Thanh HH, Thorson A. Strong propensity for HIV transmission among men who have sex with men in Vietnam: behavioural data and sexual network modelling. BMJ Open (2014) 4(1):e003526. doi:10.1136/ bmjopen-2013-003526

56. Garcia MC, Duong QL, Meyer SB, Ward PR. Multiple and concurrent sexual partnerships among men who have sex with men in Viet Nam: results from a National Internet-based Cross-sectional Survey. Health Promot Int (2016) 31(1):133-43. doi:10.1093/heapro/dau097
57. Oldenburg CE, Perez-Brumer AG, Reisner SL, Mimiaga MJ. Transactional sex and the HIV epidemic among men who have sex with men (MSM) results from a systematic review and meta-analysis. AIDS Behav (2015) 19(12):2177-83. doi:10.1007/s10461-015-1010-5

Conflict of Interest Statement: The authors declare that the research was conducted in the absence of any commercial or financial relationships that could be construed as a potential conflict of interest.

The reviewer ZW and handling Editor declared their shared affiliation, and the handling Editor states that the process nevertheless met the standards of a fair and objective review.

Copyright $\odot 2016$ Vu, Holt, Phan, La, Tran, Doan and de Wit. This is an open-access article distributed under the terms of the Creative Commons Attribution License (CC $B Y)$. The use, distribution or reproduction in other forums is permitted, provided the original author(s) or licensor are credited and that the original publication in this journal is cited, in accordance with accepted academic practice. No use, distribution or reproduction is permitted which does not comply with these terms. 